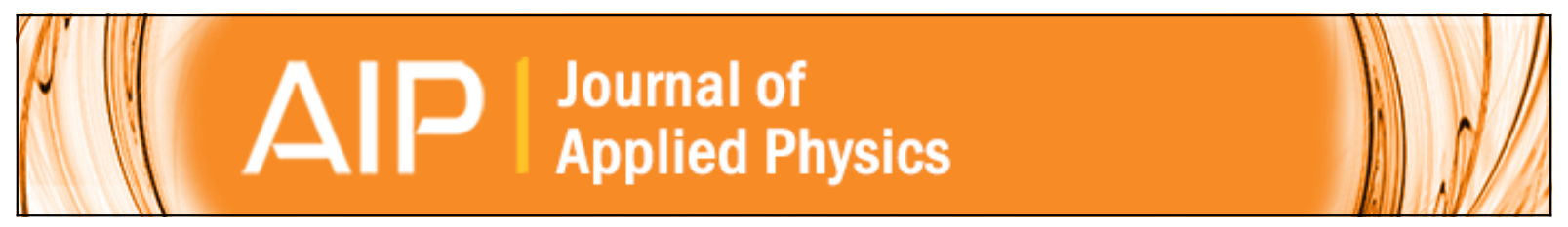

\title{
Dimensionally gradient magnetoelectric bimorph structure exhibiting wide frequency and magnetic dc bias operating range
}

Chee-Sung Park, Cheol-Woo Ahn, Su-Chul Yang, and Shashank Priya

Citation: Journal of Applied Physics 106, 114101 (2009); doi: 10.1063/1.3253739

View online: http://dx.doi.org/10.1063/1.3253739

View Table of Contents: http://scitation.aip.org/content/aip/journal/jap/106/11?ver=pdfcov

Published by the AIP Publishing

\section{Articles you may be interested in}

Influence of metglas layer on nonlinear magnetoelectric effect for magnetic field detection by frequency modulation

J. Appl. Phys. 117, 024104 (2015); 10.1063/1.4905622

Ring-type electric current sensor based on ring-shaped magnetoelectric laminate of epoxy-bonded Tb 0.3 Dy 0.7 Fe 1.92 short-fiber/NdFeB magnet magnetostrictive composite and $\mathrm{Pb}(\mathrm{Zr}$, Ti)O 3 piezoelectric ceramic J. Appl. Phys. 107, 09D918 (2010); 10.1063/1.3360349

Circumferential-mode, quasi-ring-type, magnetoelectric laminate composite-a highly sensitive electric current andor vortex magnetic field sensor

Appl. Phys. Lett. 86, 182506 (2005); 10.1063/1.1923184

Extremely low frequency response of magnetoelectric multilayer composites

Appl. Phys. Lett. 86, 102901 (2005); 10.1063/1.1881784

Vortex magnetic field sensor based on ring-type magnetoelectric laminate

Appl. Phys. Lett. 85, 2307 (2004); 10.1063/1.1791732

\section{MIT LINCOLN} LABORATORY CAREERS

Discover the satisfaction of innovation and service to the nation
- Space Control

- Air \& Missile Defense

- Communications Systems \& Cyber Security

- Intelligence, Surveillance and

Reconnaissance Systems

- Advanced
Electronics
- Tactical Systems
- Homeland
Protection
- Air Traffic Control

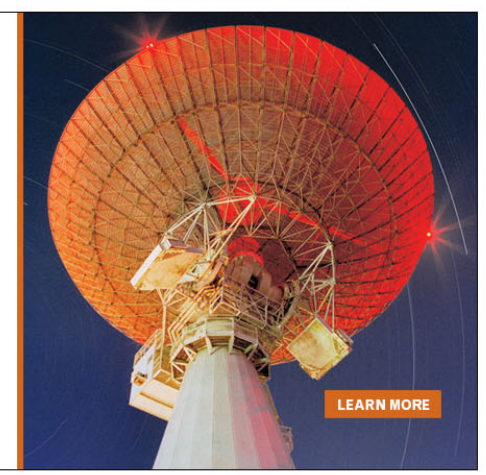




\title{
Dimensionally gradient magnetoelectric bimorph structure exhibiting wide frequency and magnetic dc bias operating range
}

\author{
Chee-Sung Park, Cheol-Woo Ahn, Su-Chul Yang, and Shashank Priya ${ }^{\text {a) }}$ \\ Center for Energy Harvesting Materials and Systems (CEHMS), Department of Materials Science and \\ Engineering, Virginia Polytechnic Institute and State University, Blacksburg, Virginia 24061, USA
}

(Received 21 July 2009; accepted 26 September 2009; published online 2 December 2009)

\begin{abstract}
We report results on a dimensionally gradient magnetoelectric (ME) sensor that demonstrates high performance over a wide frequency range and a magnetic dc bias operating in the longitudinal-transversal mode. The design of the sensor is based on a piezoelectric bimorph structure and utilizes a laminate configuration with $\mathrm{Pb}\left(\mathrm{Zn}_{1 / 3} \mathrm{Nb}_{2 / 3}\right)_{0.2}\left(\mathrm{Zr}_{0.5} \mathrm{Ti}_{0.5}\right)_{0.8} \mathrm{O}_{3}$ and Metglas as material layers. The wide-band behavior was characterized by a flat $\mathrm{ME}$ response over a wide range of magnetic dc biases corresponding to $60-215$ Oe and frequencies corresponding to $7-22 \mathrm{kHz}$. By using tip mass, the wide-band frequency response was shifted to a lower frequency range of 5-14 $\mathrm{kHz}$. The results show that the operating frequency range of the sensor can be easily shifted by changing the tip mass at the end of the composite. () 2009 American Institute of Physics. [doi:10.1063/1.3253739]
\end{abstract}

\section{INTRODUCTION}

Magnetoelectric effect (ME) has been widely investigated in composite structures consisting of magnetostrictive and piezoelectric materials. In these composites, the ME coefficient is dependent upon the elastic coupling that occurs at the interface between piezoelectric and magnetostrictive phases. ${ }^{1-5}$ The following experimental observations have been made on ME composites, which have been confirmed by theoretical modeling: (i) Magnetostrictive-piezoelectric laminate composites with a 2-2 connectivity provide much higher ME coefficients than that of single-phase materials or particulate composites. $^{2-5}$ (ii) All the composites show an enhanced ME coefficient at the electromechanical resonance (EMR) frequency. ${ }^{6,7}$ (iii) A high ME coefficient near the EMR is characterized by a sharp peak with a very narrow range of magnetic dc biases. ${ }^{6-9}$ In conjunction with the fact that EMR in laminate composites is dependent upon the dimensions, this last observation has limited the application of ME composites in magnetic field sensing; i.e., large dimensions are required to achieve a low EMR frequency. The most common mode utilized in sensing is the longitudinaltransversal mode (LT). In the LT mode, one would like to achieve a low EMR frequency and a wide operating range of frequencies and magnetic dc biases with smaller overall dimensions.

Yu et al. ${ }^{10}$ demonstrated the wide-band ME response by combining several Terfenol-D/epoxy- $\mathrm{Pb}(\mathrm{Zr}, \mathrm{Ti}) \mathrm{O}_{3}$ bilayers in parallel and series electrical connections. This approach has obvious problems: (i) Several laminate composites are required with varying EMR frequency, which increases the overall dimensions. (ii) The overall magnitude of the ME coefficient at the EMR frequency decreases significantly. (iii) The magnitude of the ME coefficient fluctuates depending upon the gradient in the external magnetic field. (iv) The

\footnotetext{
a) Author to whom correspondence should be addressed. Electronic mail: spriya@vt.edu.
}

operational frequency is still quite high. It is well known that by combining the piezoelectric element having capacitance $C_{p}$, in parallel with a shunt having capacitance $C_{s}$, the total capacitance of the system changes to $C=C_{p}+C_{s}$, which affects the natural resonance frequency $(\omega)$ given as ${ }^{11}$

$$
\omega=\sqrt{\frac{K_{\mathrm{eff}}+C^{-1} d^{2}}{m_{\mathrm{eff}}}},
$$

where $K_{\text {eff }}$ is the equivalent stiffness of the cantilever beam, $m_{\text {eff }}$ is the effective mass, and $d$ is the electromechanical coupling. Charnegie showed that for a three layer structure consisting of two piezoelectric beams bonded onto a substrate which is nonpiezoelectric (three layer laminate), the EMR frequency of one piezoelectric layer with capacitance $C_{p}$ can be shifted by changing the capacitance of the other piezoelectric layer $C_{s}$ through the expression ${ }^{12}$

$$
\omega=\sqrt{\frac{3\left(s_{11}-\frac{d_{31}^{2} A}{t\left(C_{p}+C_{s}\right)}\right)^{-1} I}{L^{3} m_{\mathrm{eff}}}},
$$

where $A$ is the area, $t$ is the thickness, $L$ is the length, $s_{11}$ is the mechanical compliance of the piezoelectric material, $d_{31}$ is the electromechanical coupling coefficient, and $I$ is the moment of inertia. Equation (2) also predicts that by combining several laminates in an electrical series and in a parallel connection, the EMR frequency can be modified. This technique has been recently utilized for energy harvesters to modify the operating frequency range. ${ }^{13}$

Our approach in achieving the wide-band behavior has been to fabricate a ME laminate structure that inherently has multiple resonance modes in close vicinity to each other. In our previous study, ${ }^{14}$ we demonstrated a near-flat ME response over a magnetic dc bias range of 90-220 Oe at a fixed frequency of $1 \mathrm{kHz}$ by synthesizing a dimensionally gradient composite structure. However, the EMR frequency of this structure was still high in the range of $20 \mathrm{kHz}$, and the ME 
coefficient exhibited a sharp peak as a function of frequency. In this study, we demonstrate a modified sensor structure with similar dimensions that reduces the resonance frequency closer to $\sim 1 \mathrm{kHz}$ and exhibits a wide-band behavior both in terms of magnetic dc bias and frequency.

\section{EXPERIMENTAL PROCEDURES}

Piezoelectric plates of composition $\mathrm{Pb}\left(\mathrm{Zn}_{1 / 3} \mathrm{Nb}_{2 / 3}\right)_{0.2}$ $\left(\mathrm{Zr}_{0.5} \mathrm{Ti}_{0.5}\right)_{0.8} \mathrm{O}_{3}$ (PZNT) were synthesized by a conventional mixed oxide method. $\mathrm{PbO}, \mathrm{ZrO}_{2}, \mathrm{TiO}_{2}, \mathrm{ZnO}$, and $\mathrm{Nb}_{2} \mathrm{O}_{5}$ powders (all 99.9\% purity from Sigma Aldrich Co., USA) were weighed and mixed in alcohol using a ball-mill with zirconia balls as the grinding media. After mixing and drying, the mixture was calcined at $850{ }^{\circ} \mathrm{C}$ for $4 \mathrm{~h}$, and the calcined powder was ball-milled again for $24 \mathrm{~h}$. Dried powders were pressed and sintered at $950{ }^{\circ} \mathrm{C}$ for $4 \mathrm{~h}$ in air. The formation of the perovskite phase in the sintered PZNT was confirmed using x-ray diffraction. No traces of any secondary phase were found in the diffraction pattern. Using scanning electron microscopy, the dense microstructure was confirmed with an average grain size of the ceramic at $\sim 2.09 \mu \mathrm{m}$.

Sintered specimens were machined to have asymmetric $\mathrm{H}$ shapes $\left(15.5 \times 5\right.$ and $9.5 \times 5 \mathrm{~mm}^{2}$ areas were connected to the bridge of $4 \times 5 \mathrm{~mm}^{2}$ ) with a thickness of $500 \mu \mathrm{m}$, electroded using silver paint (DuPont 7713 conductor paste, firing temperature of $650{ }^{\circ} \mathrm{C}$ ), and poled at $4 \mathrm{kV} / \mathrm{mm}$. The piezoelectric constant of poled PZNT plates was found to be $500 \mathrm{pC} / \mathrm{N}$, and the dielectric constant was 2219 at $1 \mathrm{kHz}$. The piezoelectric voltage constant $\left(g_{33}\right)$ was of the order of $23.41 \times 10^{-3} \mathrm{~V} \mathrm{~m} / \mathrm{N}$. The ME sensor had a bimorph configuration where two piezoelectric plates with opposite poling directions were bonded together, as shown in Fig. 1(a). On these bimorph PZNT plates, $25-\mu$ m-thick Metglas (2605SA1, Metglas Inc., USA) sheets of desired dimensions were attached using epoxy (West System, USA) with a curing temperature of $80{ }^{\circ} \mathrm{C}$. For a ME voltage coefficient measurement in the LT mode configuration, an electromagnet was used to apply the dc magnetic field, and the samples were placed in the center of the Helmholtz coil under an ac magnetic field $\left(\mathrm{H}_{\mathrm{ac}}\right)$. The voltage induced on the laminate was monitored using a lock-in amplifier. The impedance spectrum of the composites was measured by an $L C R$ meter (HP4194A, USA).

\section{RESULTS AND DISCUSSION}

The asymmetric $H$-shaped bimorph exhibited multiple resonances below $100 \mathrm{kHz}$, as shown in Fig. 1(b). The impedance and phase spectra showed the resonance peaks at $10.09,13.08,23.32,33.31,43.05,58.54$, and $82.26 \mathrm{kHz}$. For comparison, we have analyzed the resonance spectrum of a $\boldsymbol{H}$-shaped single layer ceramic plate with dimensions shown in Fig. 1(a) using a commercialized finite element modelling (FEM) code (ATILA). Hexahedral meshes were used for simulating the $\mathrm{H}$-shaped simple structure with a driving voltage of $10 \mathrm{~V}_{\text {p.p. }}$, and no constraint was applied on the geometry. The FEM results showed EMRs occurring at 40, 70, and $94 \mathrm{kHz}$, as shown in Fig. 1(c). The first mode at $40 \mathrm{kHz}$ was associated with the bending of the larger rectangular section. (a)
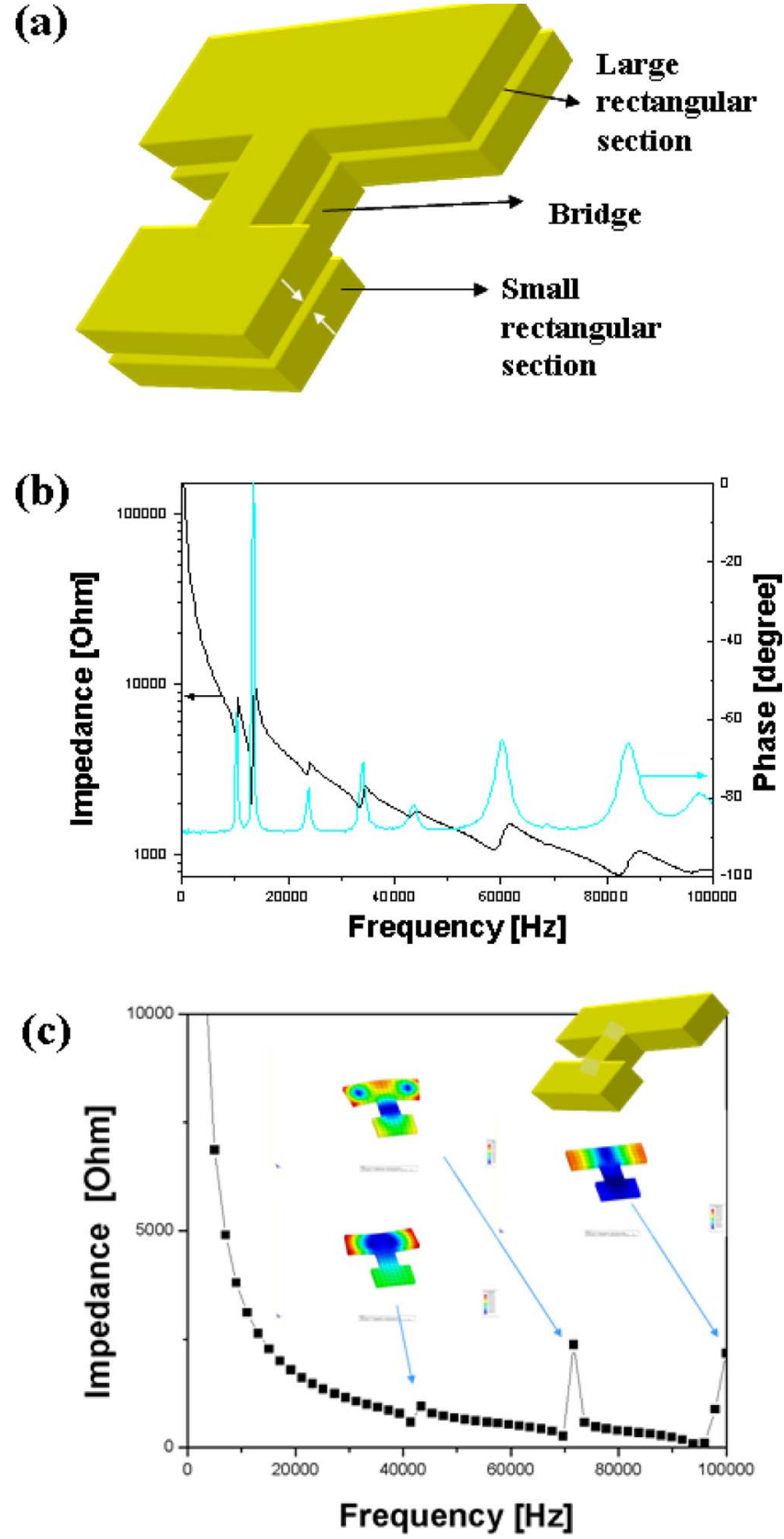

FIG. 1. (Color online) (a) Schematic diagram of an asymmetric $H$-shaped bimorph with opposite poling directions in piezoelectric layers. (b) Impedance and phase spectra for the asymmetric $\boldsymbol{H}$-shaped bimorph. (c) Impedance spectrum and FEM analysis for the asymmetric $\boldsymbol{H}$-shaped plate using ATILA software.

The second mode at $70 \mathrm{kHz}$ was found to be from the bending of two rectangular sections. The third mode at $94 \mathrm{kHz}$ was related to the combined lateral responses from the large rectangular section. On comparison, we conclude that peaks at $10.09,13.08,23.32,33.31,43.05,58.54$, and $82.26 \mathrm{kHz}$ are not related to these modes and are the result of the bimorph configuration, which indicates coupling between the two piezoelectric plates. The main peak for the bimorph was observed at $13.08 \mathrm{kHz}$. The presence of these additional peaks allows us to merge them in the desired operating range, resulting in a wide-band response.

Next, on this PZNT bimorph, 4 layers of Metglas with an area of $15 \times 7 \mathrm{~mm}^{2}$ were attached at the smaller section, 
(a)

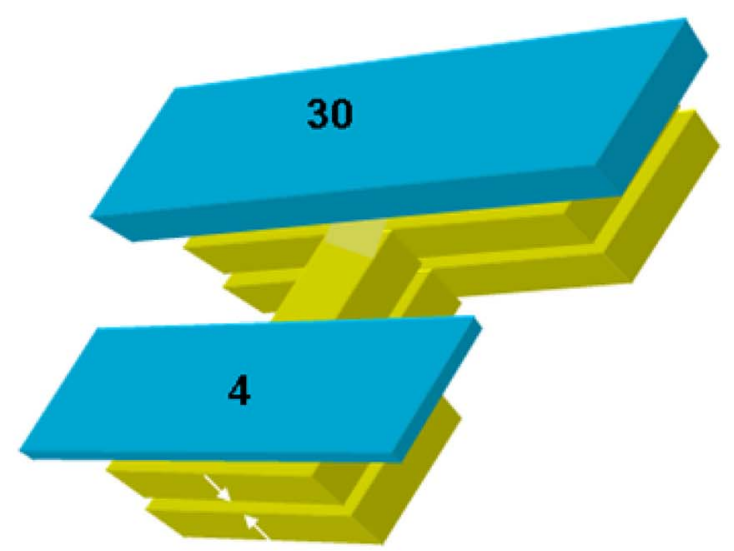

(c)

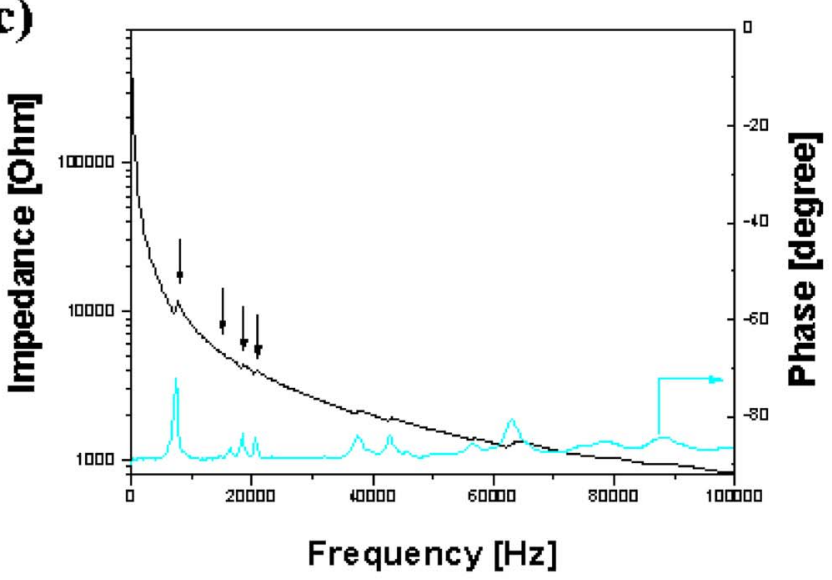

(b)

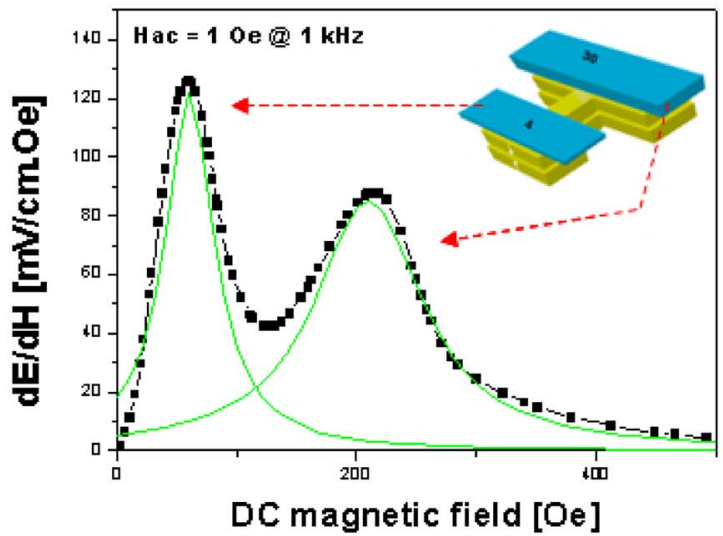

(d)

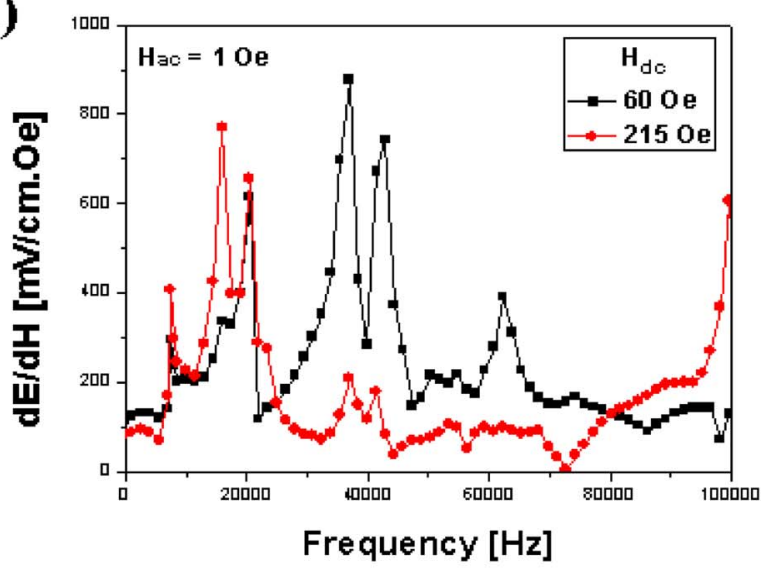

(e)

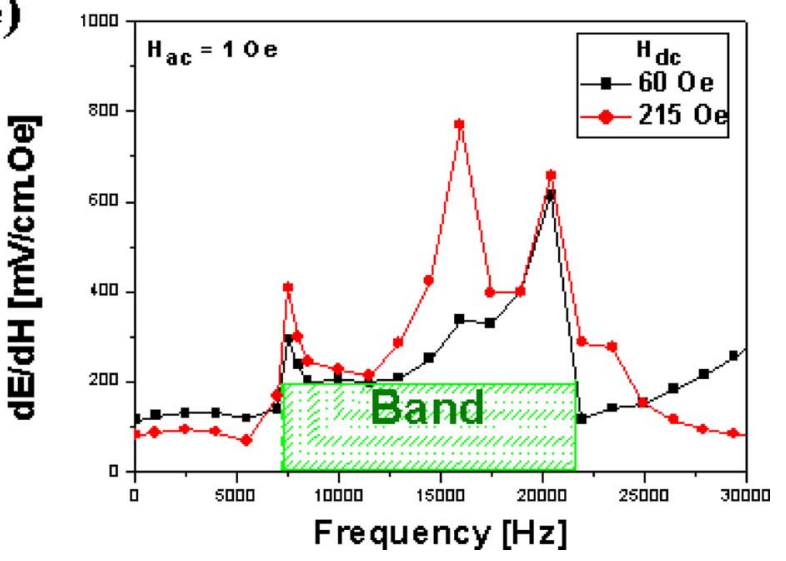

FIG. 2. (Color online) (a) Inset: schematic diagram of Metglas attached to the bimorph. (b) ME sensitivity as a function of the magnetic dc bias at a frequency of $1 \mathrm{kHz}$ and an applied ac magnetic field of $\mathrm{H}_{\mathrm{ac}}=1$ Oe. (c) Impedance and phase spectra as a function of frequency. (d) ME coefficient as a function of frequency until $100 \mathrm{kHz}$. (e) ME coefficient as a function of frequency until $30 \mathrm{kHz}$.

and 30 layers of Metglas with an area of $20 \times 7 \mathrm{~mm}^{2}$ were attached at the larger section, as shown in Fig. 2(a). There are two variables here that can be adjusted to achieve an averaging of the ME response from two sections of the $H$-laminate. First, if the area of the two sections is the same than the section which has smaller number of Metglas layers will show higher ME coefficient. Second, if the number of Metglas layers is similar to one with a smaller area, a smaller ME coefficient is shown. ${ }^{14}$ Thus, by adjusting the ratio of Metglas layers to the area of the piezoelectric rectangular sections, an average response can be obtained from the
$\mathrm{H}$-shaped structure. Figure 2(b) shows the measured ME response from the bimorph composite structure as a function of the magnetic dc bias at a frequency of $1 \mathrm{kHz}$ with a $1 \mathrm{Oe}$ applied ac field. The peak at 60 Oe was associated with the four layer Metglas section on the smaller piezoelectric surface area. The second peak of a ME coefficient at 215 Oe was associated with the 30 layer Metglas section on the larger piezoelectric area. The impedance and phase angle spectra for this laminate are shown in Fig. 2(c). After Metglas was attached on the PZNT plate, the intensity of resonances became smaller, which can be explained by the 
(a)

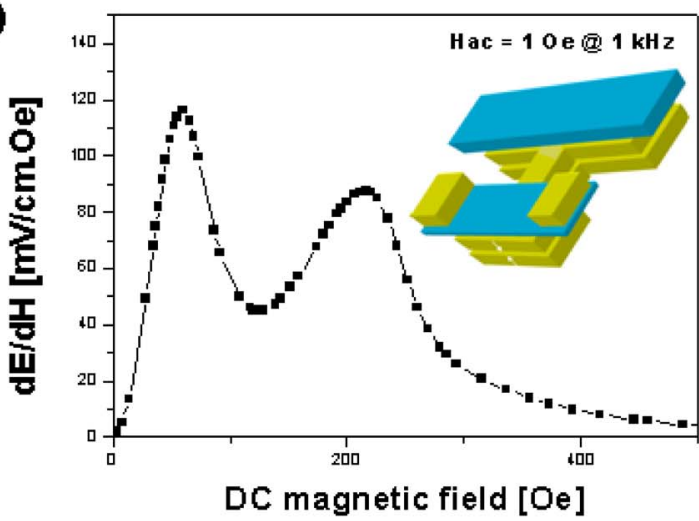

(b)

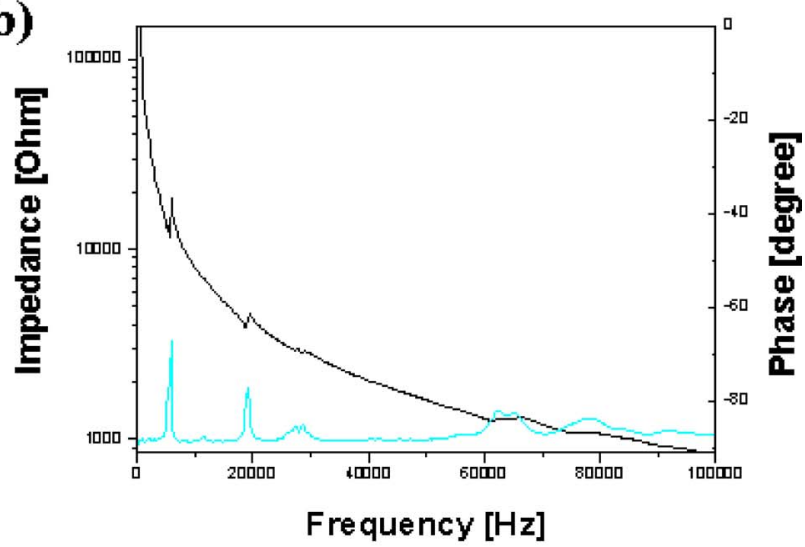

(c)

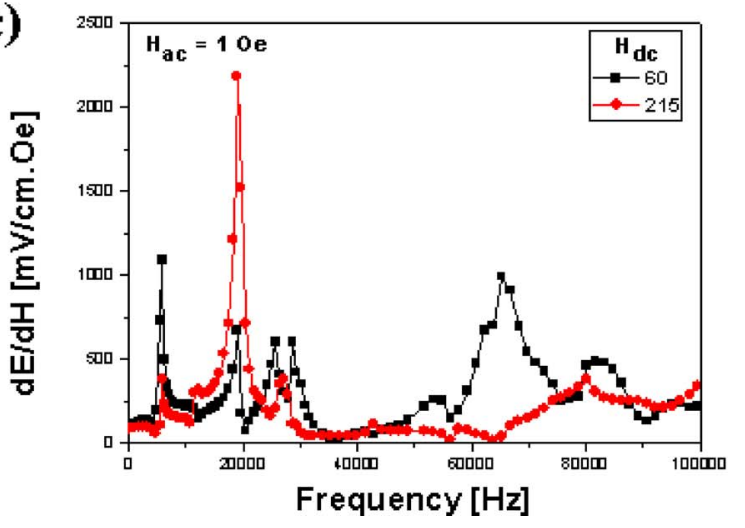

FIG. 3. (Color online) (a) ME coefficient of the laminate composite with tip mass on the smaller rectangular area as a function of a magnetic dc bias at $1 \mathrm{kHz}$. (b) Impedance and phase spectra as a function of frequency. (c) ME sensitivity as a function of frequency.

dampening effect, and the position of peaks shifted toward lower frequencies, as shown in Fig. 2(c). The weight of Metglas on PZNT plates also brought the four peaks initially found at 7.3, 16.3, 18.3, and $20.5 \mathrm{kHz}$, which originated from the peaks at $10.09,13.08,23.32,33.81$, and $43.05 \mathrm{kHz}$ in Fig. 1(b), closer to each other. Specifically, the peak at 7.3 $\mathrm{kHz}$ in Fig. 2(c) was combined with peaks at 10.09 and $13.08 \mathrm{kHz}$ in Fig. 1(b). As a result, a wide-band was formed in the frequency range of 7-22 kHz, as shown in Figs. 2(d) and 2(e). This band comprises of peaks in ME coefficient at 7.5, 15.95, and $20.43 \mathrm{kHz}$ at a magnetic dc bias of 60 Oe. Under a magnetic dc bias of $215 \mathrm{Oe}$, the peaks occur at 7.5 and $20.43 \mathrm{kHz}$. This indicates that the peak at $15.95 \mathrm{kHz}$ was (a)

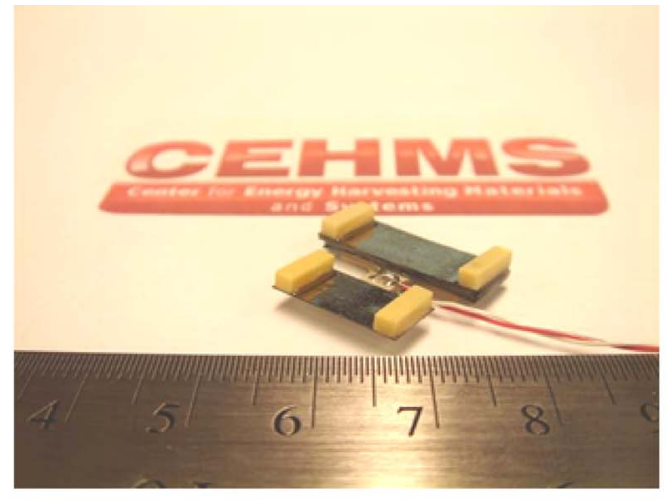

(b)

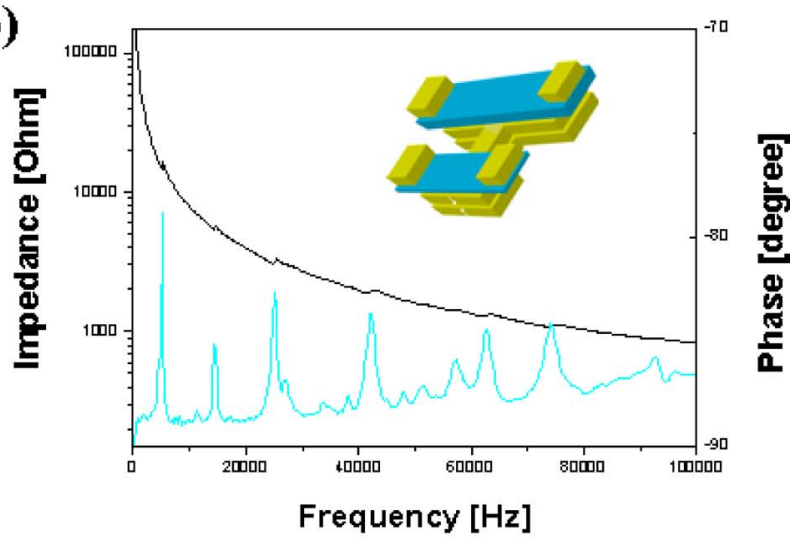

(c)

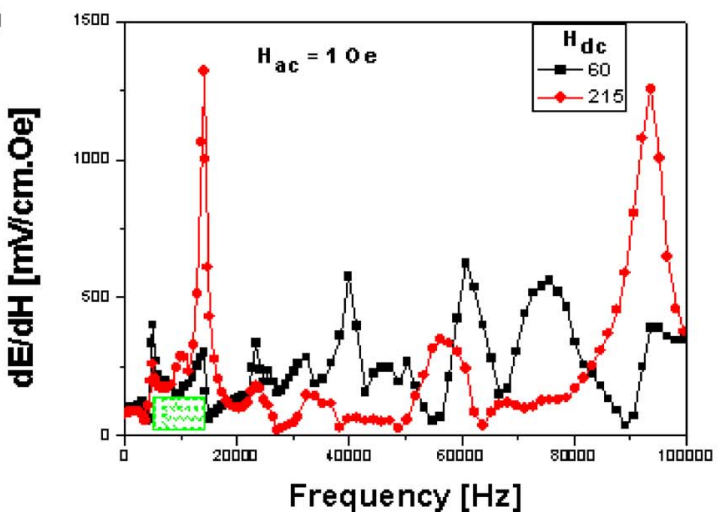

FIG. 4. (Color online) (a) Picture of the fabricated ME composite with tip mass on both smaller and larger rectangular sections. (b) Impedance and phase spectra. (c) ME sensitivity as a function of frequency.

related to magnetomechanical coupling and not to electromechanical coupling. The ME sensitivity of the sensor in this band was measured to be higher than $200 \mathrm{mV} / \mathrm{cm}$ Oe independent of the magnitude of the applied magnetic dc bias.

In order to further reduce the magnitude of the resonance frequency, the laminate composite was loaded with a tip mass. Using Eq. (1), it can be easily seen that this will result in the lowering of the resonance frequency. A tip mass of 0.2 $\mathrm{g}$ was placed on both edges of the smaller rectangular area, as shown in the schematic of Fig. 3(a). This figure also shows the effect of the tip mass on the ME response as a function of the magnetic dc bias at $1 \mathrm{kHz}$. There was not much change in the ME response compared to that in Fig. 2(b) at $1 \mathrm{kHz}$. However, significant changes were found to 
occur in the ME response as a function of frequency where the intensity of peaks in impedance spectrum increased, as shown in Fig. 3(b), and correspondingly peaks were observed in the ME spectrum shown in Fig. 3(c). The magnitude of the ME coefficient under a 60 Oe magnetic dc bias and a $5.9 \mathrm{kHz}$ frequency was found to be $1092 \mathrm{mV} / \mathrm{cm} \mathrm{Oe}$, and that under a 215 Oe magnetic dc bias and an $18.9 \mathrm{kHz}$ frequency was found to be $2184 \mathrm{mV} / \mathrm{cm}$ Oe. Compared with Fig. 2(b), this is a large increase in ME response. Further, it is interesting to note that the peaks in the ME coefficient are at different positions under different magnetic dc biases, which may be correlated with the frequency dependence of the piezomagnetic coefficient. In terms of a wide-band response, the tip mass did not result in an improvement.

We next modified the design of the sensor in Fig. 3(a) by adding the tip mass of $0.2 \mathrm{~g}$ on the edges of a larger rectangular area, as shown in Fig. 4(a). The first resonance in this case was shifted to $\sim 5 \mathrm{kHz}$, and the numbers of peaks in the impedance spectrum increased, as shown in Fig. 4(b). A wide-band $\mathrm{ME}$ response was obtained in the frequency range of 5-14 kHz with a near-flat magnitude of the ME coefficient in the range of $\sim 150 \mathrm{mV} / \mathrm{cm}$ Oe, as shown in Fig. 4(c). The results in Figs. 2-4 clearly show that by using tip mass, the resonance behavior can be shifted to lower frequency ranges with a wide-band response. These results are quite promising for practical applications such as current probe and magnetic field sensing.

\section{CONCLUSIONS}

In summary, we demonstrate a wide-band ME sensor by fabricating a dimensionally gradient bimorph structure and by combining it with a laminate configuration. Using tip masses at the ends of the sensor, we were able to obtain a wide-band frequency response in the range of $5-14 \mathrm{kHz}$ where the ME coefficient was higher than $150 \mathrm{mV} / \mathrm{cm}$ Oe and independent of the magnetic dc bias in the range of 60-215 Oe.

\section{ACKNOWLEDGMENTS}

This work was financially supported by the U.S. Army Research Office (Grant No. 47576MS).

${ }^{1}$ N. A. Spaldin and M. Fiebig, Science 309, 391 (2005).

${ }^{2}$ Y. Lin, N. Cai, J. Zhai, G. Liu, and C.-W. Nan, Phys. Rev. B 72, 012405 (2005).

${ }^{3}$ J. Ryu, A. V. Carazo, K. Uchino, and H.-E. Kim, Jpn. J. Appl. Phys., Part 1 40, 4948 (2001)

${ }^{4}$ J. Ryu, S. Priya, K. Uchino, and H.-E. Kim, J. Electroceram. 8, 107 (2002).

${ }^{5}$ S. Dong, J.-F. Li, and D. Viehland, IEEE Trans. Ultrason. Ferroelectr. Freq. Control 50, 1253 (2003).

${ }^{6}$ D. V. Chashin, Y. K. Fetisov, K. E. Kamentsev, and G. Srinivasan, Appl. Phys. Lett. 92, 102511 (2008).

${ }^{7}$ S. Dong, J. Cheng, J.-F. Li, and D. Viehland, Appl. Phys. Lett. 83, 4812 (2003).

${ }^{8}$ M. I. Bichurin, D. A. Filippov, V. M. Petrov, V. M. Laletsin, N. Paddubnaya, and G. Srinivasan, Phys. Rev. B 68, 132408 (2003).

${ }^{9}$ Y. X. Liu, J. G. Wan, J.-M. Liu, and C. W. Nan, J. Appl. Phys. 94, 5111 (2003).

${ }^{10}$ H. Yu, M. Zeng, Y. Wang, J. G. Wan, and J.-M. Liu, Appl. Phys. Lett. 86, 032508 (2005)

${ }^{11}$ M. G. Muriuki, Ph.D. thesis, University of Pittsburgh, 2004.

${ }^{12}$ D. Charnegie, M.S. thesis, University of Pittsburgh, 2005.

${ }^{13}$ H. Xue, J. Yang, and Y. Hu, IEEE Trans. Ultrason. Ferroelectr. Freq. Control 55, 1632 (2008).

${ }^{14}$ C.-S. Park, C.-W. Ahn, J. Ryu, W.-H. Yoon, D.-S. Park, H.-E. Kim, and S. Priya, J. Appl. Phys. 105, 094111 (2009). 